\title{
ULTRA-WIDEBAND METAMATERIAL FILTER BASED ON ELECTROINDUCTIVE-WAVE COUPLING BETWEEN MICROSTRIPS
}

\author{
M. Navarro-Cía, J. M. Carrasco, M. Beruete, and F. Falcone \\ Millimeter and Terahertz Waves Laboratory \\ Universidad Pública de Navarra \\ Campus Arrosadía, Pamplona 31006, Spain
}

\begin{abstract}
In this work, we analyse the frequency response of microstrip lines coupled by complementary split ring resonators (CSRRs) etched on the ground plane supporting electroinductive waves (EIWs). The single-particle configurations demonstrate the principle of operation whose bandwidths reach $67 \%$ of the central frequency. A double configuration is afterwards investigated as a further improvement of the filtering response, such as the level of the spurious lower frequency band. Finally, an ultimate prototype comprising different CSRRs along the access line, together with the aforementioned EIW-coupling is proposed for filtering undesired higher bands. Experimental results confirm numerical analysis.
\end{abstract}

\section{INTRODUCTION}

The design of microwave planar filters and couplers has taken a great advantage of the new perspective brought along by the advent of metamaterials [1]. In particular, Split Ring Resonators (SRRs) [2] and CSRRs [3] have become powerful elements in planar technology as a result of their tailored frequency response as well as their subwavelength dimensions [4].

Recently, the underlying physics of an isolated SRR or CSRR and the interaction between SRRs or CSRRs and other elements, such as series interdigitated capacitive gaps [5] and electromagnetic bandgaps [6], have been deeply studied in planar technology leading to exotic electromagnetic behaviors such as left-handed propagation [4].

Corresponding author: M. Navarro-Cía (miguel.navarro@unavarra.es). 
Among other consequences, EIWs are supported by CSRRs because of the close interaction between neighbor elements $[7,8]$. Therefore, in a discontinuous microstrip that otherwise exhibits stop-band at certain frequencies, a passband can be achieved at those frequencies if CSRRs are etched on the ground plane, being propagation supported by the existence of the CSRRs [7]. This approach is employed in this paper in order to design coupled microstrip filters.

Subsequently, we further investigate the viability of coupling microstrip lines within an enhanced ultra-wide bandwidth with filtering response. The strategy used to improve the coupling bandwidth and minimize the spurious bands arising at lower and higher frequencies is to combine two electro-inductive coupling branches, i.e., two stages in cascade. This leads to $70 \%$ measured bandwidth of the central frequency and the spurious lower frequency lobe of $-30 \mathrm{~dB}$. Finally, chains of CSRRs are designed to be placed underneath the access microstrip lines in order to remove the undesired higher frequency spurious band, taking advantage of the frequency selective nature of the CSRR elements.

\section{DESIGN AND FABRICATION OF THE ELECTRO-INDUCTIVE PARTICLE}

A schematic with the relevant dimensions of the single-CSRR configuration, as well as the fabricated prototypes, is presented in Fig. 1. They consist of two microstrip lines with a separation between line of $d_{x}=1 \mathrm{~mm}$ to each other and an overlap region $2 d_{z}$. Within the overlap area, that is, at the end of each microstrip, a CSRR is etched on the ground plane. The relevant dimensions of the microstrip lines and CSRRs are summarized in Table 1.

Table 1. Parameters of the single scheme (in $\mathrm{mm}$ ).

\begin{tabular}{|c|c|c|}
\hline Parameters & Prototype 1 & Prototype 2 \\
\hline$L_{x}$ & 5 & 6 \\
\hline$L_{z}$ & 4 & 6 \\
\hline$w$ & 1.2 & 1.2 \\
\hline$c$ & 0.5 & 0.3 \\
\hline$d$ & 0.1 & 0.3 \\
\hline$d_{x}$ & 1 & 1 \\
\hline$d_{z}$ & 2 & 3.25 \\
\hline
\end{tabular}

Reproduced courtesy of The Electromagnetics Academy 


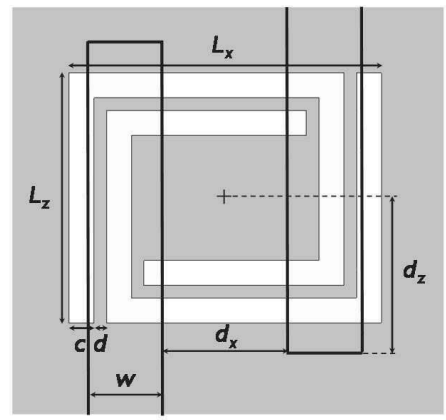

(a)

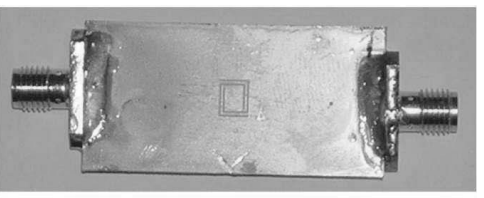

(b)

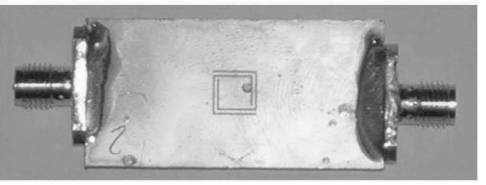

(c)

Figure 1. (a) Schematic of the single topology with the relevant parameters. The microstrip lines are over-imposed to the CSRR. Bottom view of prototype 1 (b) and prototype 2 (c). Reproduced courtesy of The Electromagnetics Academy

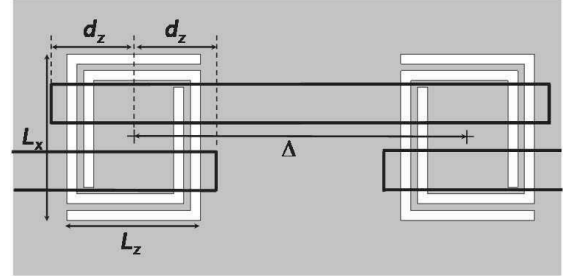

(a)

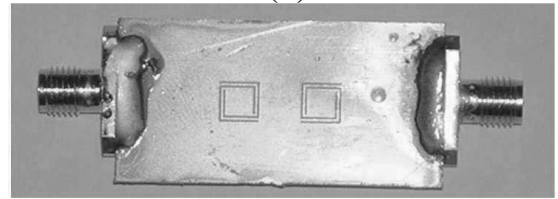

(b)

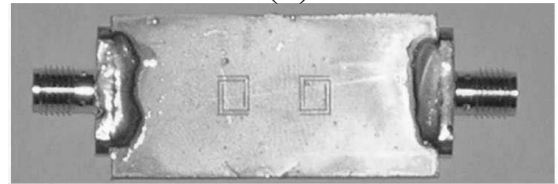

(c)

Figure 2. (a) Schematic of the double topology with the relevant parameters. Bottom view of prototype 1 (b) and prototype 2 (c). Reproduced courtesy of The Electromagnetics Academy

On the other hand, the double topology, whose sketch and picture are shown in Fig. 2, presents three microstrip lines: one is split into two branches and adjacent to them at a distance $d_{x}=0.8 \mathrm{~mm}$ and $0.4 \mathrm{~mm}$, for the first and second prototype respectively, a conductor strip which not only covers the gap region, but also overlaps slightly 
Table 2. Parameters of the double scheme (in $\mathrm{mm}$ ).

\begin{tabular}{|c|c|c|}
\hline Parameters & Prototype 1 & Prototype 2 \\
\hline$L_{x}$ & 5 & 5 \\
\hline$L_{z}$ & 4 & 5 \\
\hline$\Delta$ & 10 & 10 \\
\hline$w$ & 1.2 & 1.2 \\
\hline$c$ & 0.3 & 0.3 \\
\hline$d$ & 0.2 & 0.2 \\
\hline$d_{x}$ & 0.4 & 0.8 \\
\hline$d_{z}$ & 2 & 3.5 \\
\hline
\end{tabular}

Reproduced courtesy of The Electromagnetics Academy

with the two aforementioned branches. The CSRRs are etched on both ends of this central microstrip. The parameters regarding this double topology are presented in Table 2. Those not shown in Fig. 2(a) have the same meaning as before.

The substrate used for the design and fabrication was Rogers RO3010 with electrical permittivity $\varepsilon_{r}=10.2$, height $h=1.27 \mathrm{~mm}$, and conductor thickness $t=0.035 \mathrm{~mm}$. The fabrication has been performed by a LPKF ProtoMat 93s numerical milling machine.

\section{NUMERICAL AND EXPERIMENTAL RESULTS}

Prior to the prototype fabrication, full wave simulation results have been obtained with the aid of the transient solver of the commercial software CST Microwave Studio ${ }^{T M}$, which is based upon Finite Integration Time Domain Method. Let us start with the single-CSRR configuration and tackle the double topology subsequently.

Figure 3 shows the simulated $S$-parameters for the proposed designs without and with CSRR at their end for prototype 1 (Figs. 3(a) and 3(b)) and prototype 2 (Figs. 3(c) and 3(d)). As shown in the figure, the coupling between microstrip lines is negligible when the CSRR is not etched. However, when a CSRR is placed underneath the end of the microstrip lines, a clear passband from $3.5 \mathrm{GHz}$ to $6.5 \mathrm{GHz}$ for prototype 1 (Fig. 3(b)) and from $2.2 \mathrm{GHz}$ to $4.4 \mathrm{GHz}$ for prototype 2 (Fig. 3(d)) can be observed. Fig. 3 also reveals that at lower and higher frequencies, spurious bands appear. It is desirable to remove the spurious bands in a final prototype. This fact will be addressed afterwards. 
Without CSRR

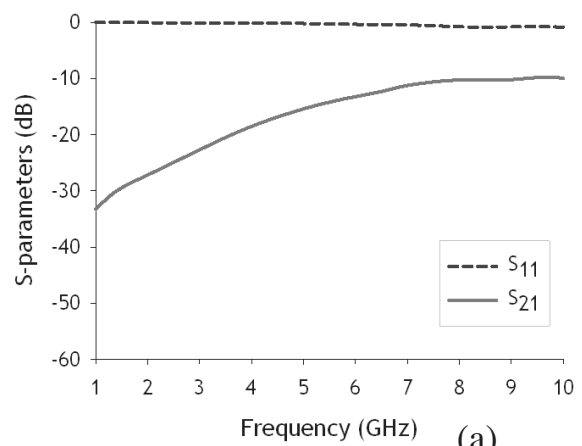

(a)

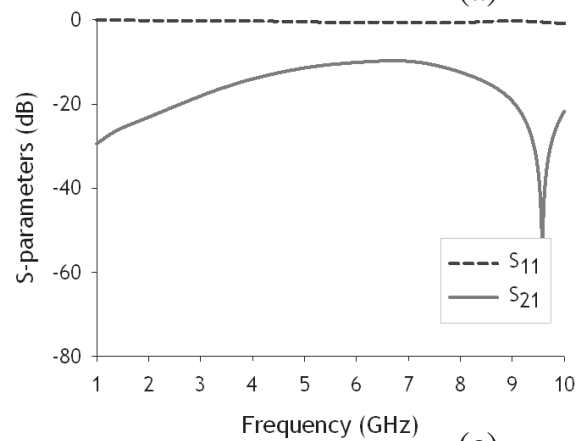

(c)
With CSRR

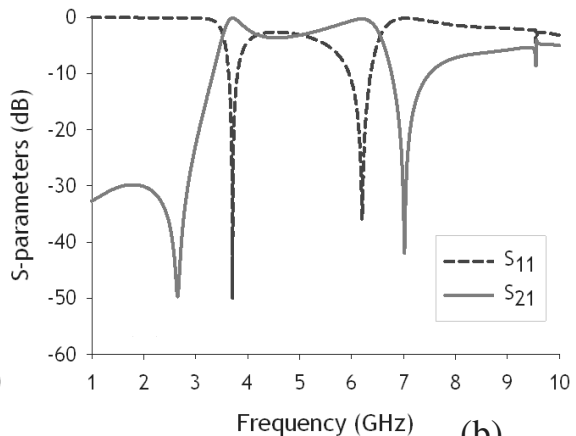

(b)

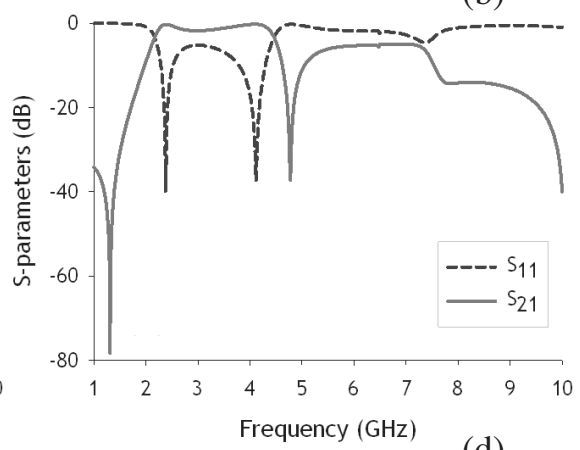

(d)

Figure 3. Simulated $S$-parameters $\left(S_{11}\right.$ and $S_{21}$ are represented in dashed blue and solid red curves) for prototype 1 (b) and prototype 2 (d). Both prototypes without the etched CSRR are shown in (a) and (c) respectively, to stress the role of the EIW-based coupling through the CSRR.

\section{Reproduced courtesy of The Electromagnetics Academy}

Figure 4 shows the simulated $S$-parameters for the proposed double-scheme designs. Once again, in order to highlight the role of the EIWs (due to the etched CSRRs), the prototypes without and with CSRRs are rendered on the left- and right-hand sides respectively. The results display similar features to the single-scheme topologies: the inclusion of CSRRs gives rise (prototype 2, see Figs. 4(c) and $4(\mathrm{~d})$ ) or even broadens (prototype 1, see Figs. 4(a) and 4(b)) the coupling passband. It is worth noting in this case that compared with single CSRR configuration, the level of the spurious band at lower frequencies decreases to values below $-45 \mathrm{~dB}$ in both cases. Therefore, the remaining concern is to remove the band observed at frequencies higher than $6 \mathrm{GHz}$. 
Without CSRRs
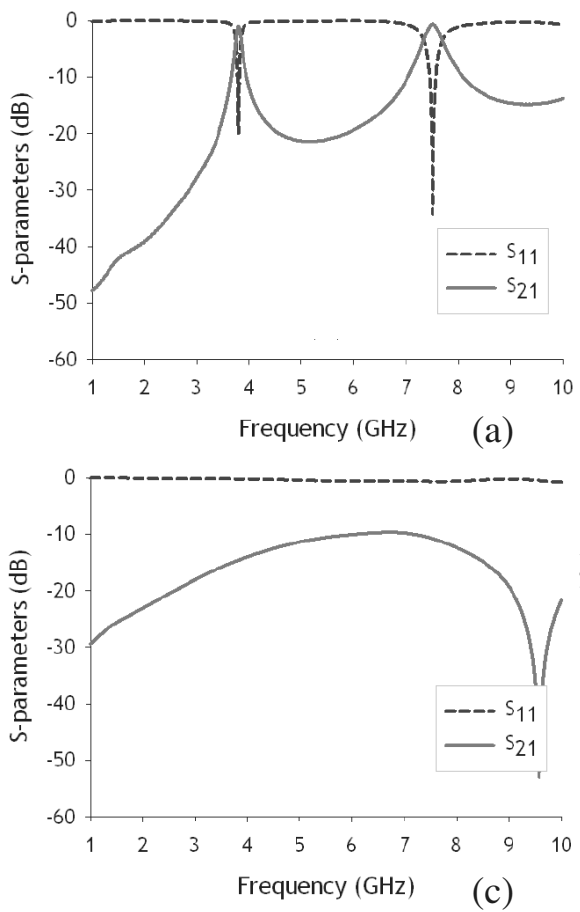
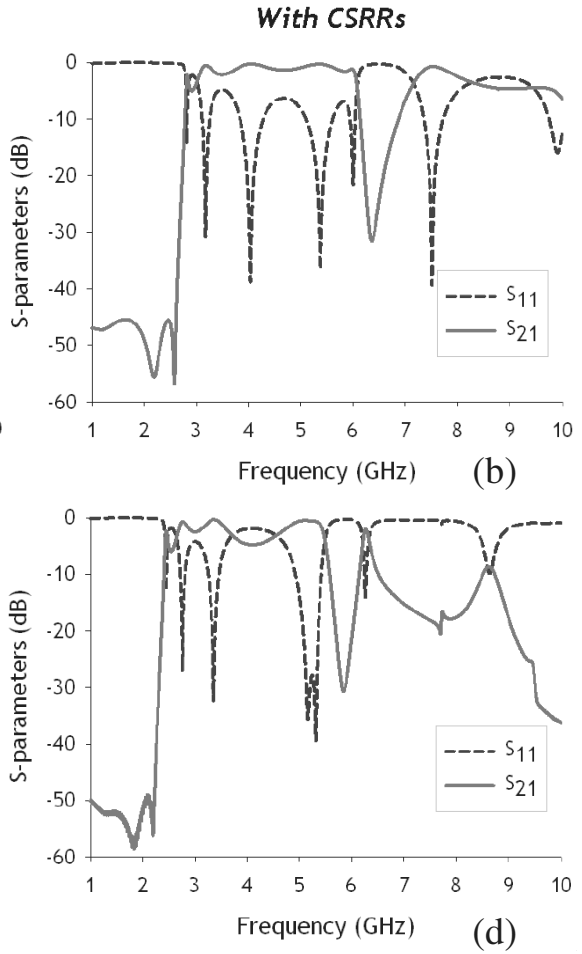

Figure 4. Simulated $S$-parameters $\left(S_{11}\right.$ and $S_{21}$ are represented in dashed blue and solid red curves respectively) for double-scheme prototype 1 (b) and prototype 2 (d). Both prototypes without the etched CSRR are shown in (a) and (c) respectively, to stress the role of the EIW-based coupling through the CSRR.

\section{Reproduced courtesy of The Electromagnetics Academy}

To finish with the numerical analysis, we plot the average surface current at one random frequency within the passband (without loss of generality) for prototypes 1 of single- (Fig. 5(a)) and doubleschemes (Fig. 5(b)). Notice that there is propagation along the whole planar transmission line, as it was expected from $S$-parameters, and an enhancement of the current density around the CSRRs on the ground plane (Notice that the average surface current is reduced at the sections of the microstrip lines above the CSRR). This reveals that the resonator is excited and supporting the EIW coupling.

Measurements have been carried out with an Agilent 8722ES VNA up to $10 \mathrm{GHz}$, using full two port $3.5 \mathrm{~mm} \mathrm{D}$ connector calibration $3.5 \mathrm{~mm}$. Figs. 6(a) and 6(b) show the $S$-parameters for the singleschemes, whereas Figs. 6(c) and 6(d) are given for the double-schemes. 


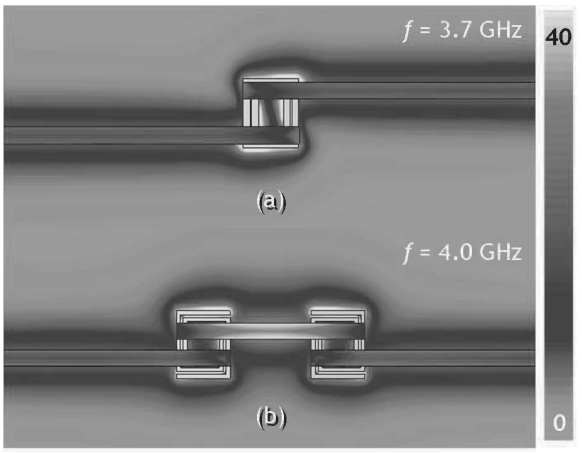

Figure 5. Average surface current $(\mathrm{A} / \mathrm{m})$ for prototypes 1 of single (a) and double topology (b).

Reproduced courtesy of The Electromagnetics Academy
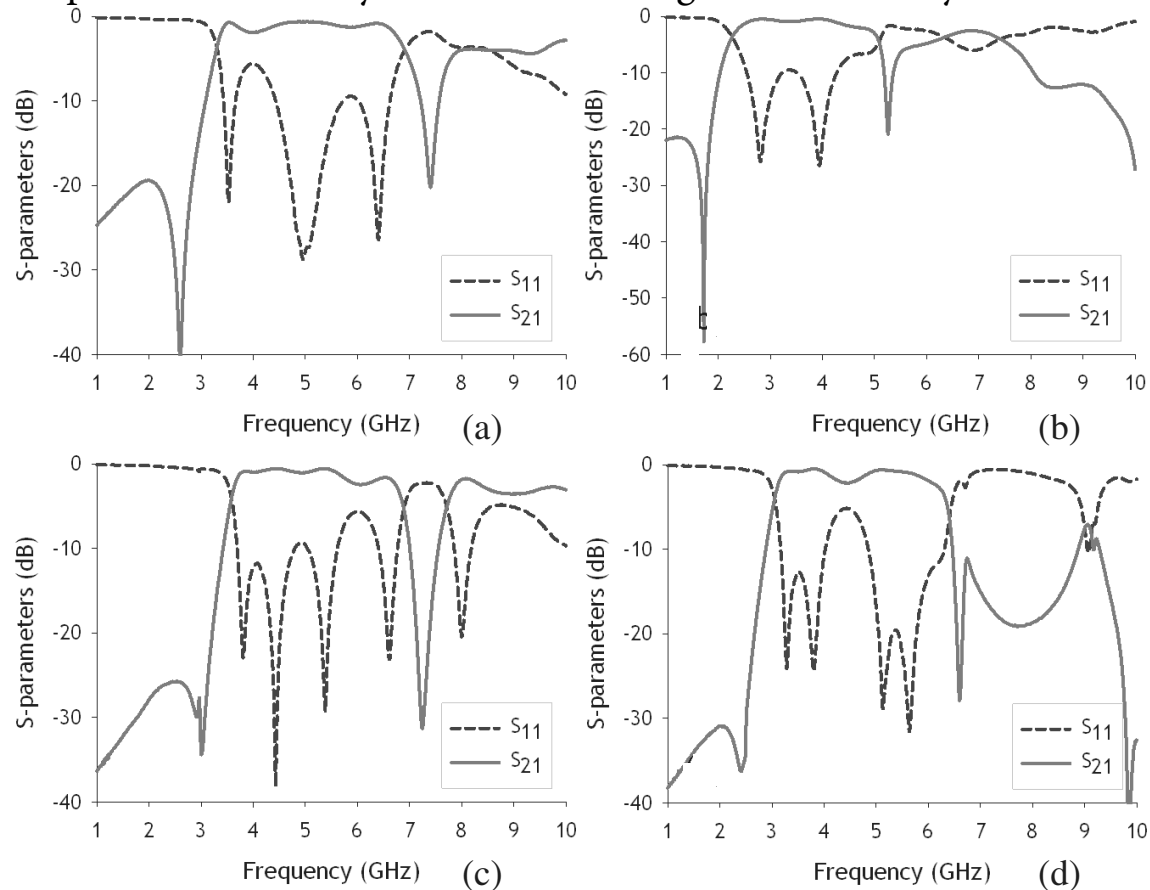

Figure 6. Measured $S$-parameters $\left(S_{11}\right.$ and $S_{21}$ are represented in dashed blue and solid red curves respectively) for single-scheme (a) and (b), and double-scheme (c) and (d): prototype 1 (a), (c), and prototype 2 (b), (d).

Reproduced courtesy of The Electromagnetics Academy 
As can be seen, there is good agreement between numerical and experimental results. In the case of single-scheme, the EIW passband extends experimentally from $3.3 \mathrm{GHz}$ to $6.8 \mathrm{GHz}$ for prototype 1 (Fig. 6(a)) and from $2.3 \mathrm{GHz}$ to $5 \mathrm{GHz}$ for prototype 2 (Fig. 6(b)), which implies a slight shift with respect to the simulation. Moreover, the passband suffers a lower ripple than predicted in simulations, which is a desired feature when one needs to fulfill specifications. The magnitude of the spurious bands is slightly higher than that in simulation due to tolerances in the fabrication process, slight changes in material parameters of the employed substrate batch and non-ideal behavior of the input/output connectors.

On the other hand, the EIW passband goes experimentally from $3.6 \mathrm{GHz}$ to $6.8 \mathrm{GHz}$ and from $3.0 \mathrm{GHz}$ to $6.3 \mathrm{GHz}$ for prototypes 1 and 2 of the double-scheme. In this case, the passband has been clearly reduced compared to the simulation, although the shape of the electromagnetic response is preserved. We are inclined to believe that this disagreement may be caused by some fabrication errors. Finally, the comments done in previous paragraph about the ripple within the passband and spurious bands can be brought about here as well.

\section{ULTIMATE PROTOTYPE}

It has been pointed out previously that it would be desirable to remove from the electromagnetic response the spurious bands that appear below and above the passband frequency range. The lower band has already been minimized by the double topology (two stages in cascade). Now, the approach to remove the higher band is to exploit the commonly used rejection response of the CSRR [4]. By placing a chain of tuned CSRRs underneath the access microstrip lines, one can design a wideband rejection band, which coincides with the undesired spurious band and thus, remove it [9]. Moreover, since the CSRR is operating in subwavelength regime, the occupied layout area by this chain of CSRRs is small.

The proposed design is rendered in Fig. 7(a). It is based on the prototype 2 of double-scheme together with a chain of CSRRs. The CSRRs have metal and air ring, as well as metal gap of $0.2 \mathrm{~mm}$. The vertical dimension of the CSRRs is $2 \mathrm{~mm}$, whereas the horizontal one ranges from $2.3 \mathrm{~mm}$ to $3.8 \mathrm{~mm}$ with a non-uniform step reached after an optimized procedure. The total length of the layout is $81 \mathrm{~mm}$.

The simulated results can be seen in Fig. 7(b). As expected, the EIW passband remains present, and the first band appearing at around 6 to $8 \mathrm{GHz}$ in preceding double-scheme prototypes has been removed clearly. 


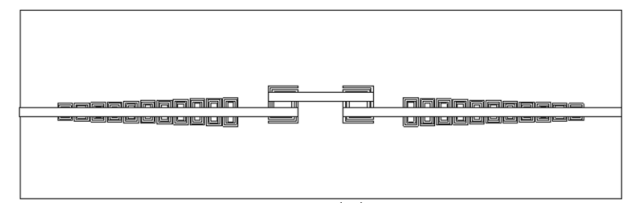

(a)

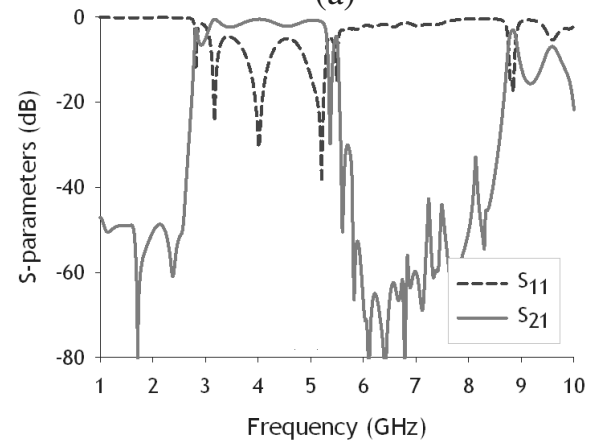

(b)

Figure 7. (a) Sketch of the proposed EIW-filter. (b) Simulated $S$ parameters $\left(S_{11}\right.$ and $S_{21}$ are represented in dashed blue and solid red curves respectively).

Reproduced courtesy of The Electromagnetics Academy

\section{CONCLUSION}

In this paper, we show that electroinductive waves are a powerful tool to design wideband filters under the perspective of metamaterials, which may find application in devices such as image rejection filters, system diplexer and triplexer, antenna duplexer-filter and coupling circuits of several types. Despite the narrowband response commonly associated with split ring resonators and its complementary particle, by combining these resonators with discontinuous microstrip lines (note that capacitive-gaps have a long story in filters design, but their response is narrower than the one achieved by our prototype, which stresses the key role played by the CSRRs-based coupled filter), wideband responses are attainable as demonstrated in this work. Moreover, because of the subwavelength character of the CSRRs, the section providing the wideband coupling is clearly smaller than, for instance, coupled-line bandpass filters. Numerical results have been confirmed experimentally in the microwave regime.

\section{ACKNOWLEDGMENT}

This work has been supported by the Spanish Government by the project TEC2008-06871-C02-01. 


\section{REFERENCES}

1. Solymar, L. and E. Shamonina, Waves in Metamaterials, Oxford University Press, New York, 2009.

2. Pendry, J. B., A. J. Holden, D. J. Robbins, and W. J. Stewart, "Magnetism from conductors and enhanced nonlinear phenomena," IEEE Trans. Microw. Theory Tech., Vol. 47, No. 11, 20752084, 1999.

3. Falcone, F., T. Lopetegi, M. A. G. Laso, J. D. Baena, J. Bonache, M. Beruete, R. Marqués, F. Martín, and M. Sorolla, "Babinet principle applied to metasurface and metamaterial design," Phys. Rev. Lett., Vol. 93, No. 12, 197491-1-197491-4, 2004.

4. Marqués, R., F. Martín, and M. Sorolla, Metamaterials with Negative Parameters: Theory, Design, and Microwave Applications, John Wiley and Sons, New York, 2008.

5. Jarauta, E., M. A. G. Laso, T. Lopetegi, F. Falcone, M. Beruete, J. D. Baena, J. Bonache, I. Gil, J. García-García, J. A. Marcotegui, F. Martín, R. Marqués, and M. Sorolla, "Novel microstrip backward coupler with metamaterial cells for fully planar fabrication techniques," Microw. Opt. Tech. Lett., Vol. 48, No. 7, 1205-1209, 2006.

6. Navarro-Cía, M., F. Falcone, M. Beruete, I. Arnedo, J. Illescas, J. A. Marcotegui, M. A. G. Laso, and T. Lopetegi, "Left-handed behaviour in a microstrip line loaded with squared split-ring resonators and an EBG pattern," Microw. Opt. Tech. Lett., Vol. 49, No. 11, 2689-2692, 2007.

7. Beruete, M., F. Falcone, M. J. Freire, R. Marqés, and J. D. Baena, "Electroinductive waves in chains of complementary metamaterial elements," Appl. Phys. Lett., Vol. 88, No. 8, 083503-1-083503-3, 2006.

8. Navarro-Cía, M., M. Beruete, S. Agrafiotis, F. Falcone, M. Sorolla, and S. A. Maier, "Broadband spoof plasmons and subwavelength electromagnetic energy confinement on ultrathin metafilms," Opt. Express, Vol. 17, No. 20, 18184-18195, 2009.

9. Martin, F., F. Falcone, J. Bonache, R. Marqués, and M. Sorolla, "Miniaturized coplanar waveguide stop band filters based on multiple tuned split ring resonators," IEEE Microw. Wirel. Comp. Lett., Vol. 13, No. 12, 511-513, 2003. 